\title{
Robust weighted expected residual minimization formulation for stochastic vector variational inequalities
}

\author{
Yong Zhao ${ }^{a}$, Zai Yun Peng ${ }^{\text {a,*, Yun Bin Zhao }}{ }^{b}$ \\ ${ }^{a}$ College of Mathematics and Statistics, Chongqing JiaoTong University, Chongqing 400074, China. \\ ${ }^{b}$ School of Mathematics, University of Birmingham, Birmingham, UK.
}

Communicated by Y. B. Xiao

\begin{abstract}
In order to deal with (stochastic) multi-objective optimization problems, a robust Pareto optimal solution by minimizing the worst case weighted sum of objectives on a given weight set is considered [J. Hu, S. Mehrotra, Oper. Res., 60 (2011), 936-953], [J. Hu, T. Homem-de-Mello, S. Mehrotra, Manuscript, (2010)]. Based on this idea, we introduce a new class of deterministic model for stochastic vector variational inequalities, called robust weighted expected residual minimization model. Then we propose sample average approximation (SAA) approach to solve robust weighted expected residual minimization problems. Some convergence results are established for the approximation problem in terms of the optimal value and the set of optimal solutions. (C) 2017 All rights reserved.
\end{abstract}

Keywords: Robust weighted expected residual minimization, stochastic vector variational inequalities, convergence. 2010 MSC: 90C33, 90C15.

\section{Introduction}

The concept of variational inequalities plays a major role in the study of both the qualitative and numerical analysis of various mathematical models. Different types of variational inequalities and their extensions have been extensively investigated, see $[1,2,4,8,10,11,15,17,27-29,32]$ and the references therein. The concept of vector variational inequality (VVI) was introduced first by Giannessi [10], which is a generalization of a scalar variational inequality (VI) to the vector case. Since then, there has been a significant number of research results on VVI; see $[1,2,4,11,15,17,32]$ and the references therein.

The scalarization approach is a popular method to solve VVI, which is transform VVI into VI by choosing a weight vector; see, e.g., $[1,2,4,15,17]$. In fact, it is hard to determine exactly a preferable weight over others for a decision maker. In this paper, inspired by recent developments on multiobjective optimization, a robust weighted model based on gap function for VVI is proposed.

Since some elements in many practical decision problems may involve uncertainties, it is significant for studying stochastic VVI (SVVI). Note that stochastic variational inequalities (SVI) have been studied

\footnotetext{
${ }^{*}$ Corresponding author Zhao)

Email addresses: zhaoyongty@126.com (Yong Zhao), pengzaiyun@126.com (Zai Yun Peng), y.zhao.2@bham.ac.uk (Yun Bin
} 
in $[3,6,7,12,16,18,19,21-24,30,31,34]$. We know that SVI does not have a common solution in general. Therefore, for the sake of getting reasonable solutions in some senses, an appropriate deterministic reformulation is needed. There are some deterministic formulations have been discussed for SVI. For example, expected value formulation [12, 16, 19,31], expected residual minimization (ERM) formulation $[3,6,7,18,21-23,34]$, conditional value-at-risk formulation [5], and worst-case residual minimization formulation [30]. Based on the equivalent representation for SVVI, Zhao et al. [33] introduced a deterministic model for SVVI, which is an extension of a series of works on ERM formulation of SVI to the vector case. In [14], Hu and Mehrotra introduced the robust weighted sum approach for multiobjective optimization problem. $\mathrm{Hu}$ et al. [13] presented and studied two models for uncertainty/stochastic multiobjective optimization problem.

Motivated by the works $[13,14]$ on (stochastic) multiobjective optimization, we introduce a new class of deterministic model for SVVI, called robust weighted ERM model. An approximation method based on SAA techniques is proposed for solving the robust weighted ERM problem. Under some assumptions, the convergence and exponential convergence rates for the optimal value and the set of optimal solutions of the approximate problem are established.

\section{Preliminary}

In what follows, $\|\cdot\|$ denotes the Euclidean norm of a vector. $\operatorname{Proj}_{S}(x)$ denotes the projection of a point $x$ onto $S$, where $S$ is a closed convex set. For given $\|\cdot\|$, denote by $d(x, C):=\inf _{x^{\prime} \in C}\left\|x-x^{\prime}\right\|$ the distance from $x$ to a set $C$. For sets $C, D \subset \mathbb{R}^{n}$, denote by $\mathbb{D}(C, D):=\sup _{x \in C} d(x, D)$ the deviation of $C$ from $D$. Furthermore, denote by

$$
\Lambda:=\left\{\lambda \in \mathbb{R}^{m}: \lambda_{j} \geqslant 0, \sum_{j=1}^{m} \lambda_{j}=1\right\} .
$$

Consider VVI: Find $x^{*} \in S$ such that

$$
\left(\left(y-x^{*}\right)^{\mathrm{T}} \mathrm{F}_{1}\left(x^{*}\right), \cdots,\left(y-x^{*}\right)^{\mathrm{T}} \mathrm{F}_{\mathrm{m}}\left(x^{*}\right)\right) \notin-i n t \mathbb{R}_{+}^{\mathrm{m}}, \quad \forall \mathrm{y} \in \mathrm{S},
$$

where $S \subset \mathbb{R}^{n}$ is a nonempty, convex and closed set and $F_{j}: \mathbb{R}^{n} \rightarrow \mathbb{R}^{n}(j=1,2, \cdots, m)$ are vector-valued functions. For abbreviation, set $F:=\left(F_{1}, \cdots, F_{m}\right)$. We use Sol $(F, S)$ to denote the solution set of VVI.

Clearly, for $m=1$, VVI collapses to VI: Find $x^{*} \in S$ such that

$$
\left(y-x^{*}\right)^{\mathrm{T}} \mathrm{F}\left(x^{*}\right) \geqslant 0, \quad \forall y \in S .
$$

In order to solve VVI, consider the scalar VI as follows: For any given $\lambda \in \Lambda$, find $x^{*} \in S$ such that

$$
\left(y-x^{*}\right)^{\mathrm{T}} \sum_{j=1}^{m} \lambda_{j} \mathrm{~F}_{j}\left(x^{*}\right) \geqslant 0, \quad \forall y \in S,
$$

and denote by $\operatorname{Sol}(\mathrm{F}, \mathrm{S})_{\lambda}$ the solution set of (2.2).

The following result shows the relationships between VVI and VI.

Theorem 2.1 ([17, Theorem 2.1]). It holds that

$$
\operatorname{Sol}(F, S)=\bigcup_{\lambda \in \Lambda} \operatorname{Sol}(F, S)_{\lambda}
$$

Following [9], for any given $\lambda \in \Lambda$, a regularized gap function is introduced for (2.2) as follows:

$$
\phi(x, \lambda):=\max _{y \in S}\left\{(x-y)^{\mathrm{T}} \sum_{j=1}^{m} \lambda_{j} F_{j}(x)-\frac{\alpha}{2}\|x-y\|^{2}\right\},
$$


where $\alpha>0$ is a given parameter. From [9], for given $\lambda \in \Lambda$ and for any $x \in S$,

$$
\phi(x, \lambda)=(x-H(x, \lambda))^{\mathrm{T}} \sum_{j=1}^{m} \lambda_{j} F_{j}(x)-\frac{\alpha}{2}\|x-H(x, \lambda)\|^{2},
$$

where

$$
H(x, \lambda):=\operatorname{Proj}_{S}\left(x-\alpha^{-1} \sum_{j=1}^{m} \lambda_{j} F_{j}(x)\right) .
$$

It has been shown that for any given $\lambda \in \Lambda$,

- $\phi(x, \lambda) \geqslant 0$ for every $x \in S$;

- for $x^{*} \in S, \phi\left(x^{*}, \lambda\right)=0$ if and only if $x^{*}$ solves (2.2).

Therefore, for given $\lambda \in \Lambda$, solving (2.2) is equivalent to solving the minimization problem

$$
\min _{x \in S} \phi(x, \lambda) .
$$

As discussed above, scalarization approach is one of the most popular approaches to deal with VVI (2.1), which transforms VVI into VI by choosing a pre-determined weight vector. In practice, it is very hard to determine exactly a preferable weight over others for a decision maker. Motivated by the works [13, 14] on (stochastic) multiobjective optimization, we introduce a robust weighted model for VVI (2.1). That is, let us consider

$$
\min _{x \in S} \max _{\lambda \in \Lambda} \phi(x, \lambda)
$$

The model (2.3) is a minimax problem, which provides a way to overcome the difficulty of choosing weights.

Denote by $\theta(x):=\max _{\lambda \in \Lambda} \phi(x, \lambda)$.

Theorem 2.2. Let $\mathrm{S}$ be a compact set and $\mathrm{F}_{i}$ be a continuous function. Then the following properties hold:

(i) $\theta(x) \geqslant 0$ for every $x \in S$;

(ii) for $x^{*} \in S, \theta\left(x^{*}\right)=0$, then $x^{*}$ solves VVI (2.1).

Proof. By setting $y=x$ in the expression of $\theta(x)$, it is not hard to obtain that $\theta(x) \geqslant 0$ for all $x \in S$. Let us consider $x^{*} \in S$ such that $\theta\left(x^{*}\right)=0$. That is, $\max _{\lambda \in \Lambda} \phi\left(x^{*}, \lambda\right)=0$. It follows from the continuity of $F_{i}$ and the compactness of $S$ that there exists $\lambda^{*} \in \Lambda$ such that $\phi\left(x^{*}, \lambda^{*}\right)=0$. Therefore, $x^{*}$ solves (2.2) with $\lambda=\lambda^{*}$. By Theorem 2.1, $x^{*}$ solves VVI (2.1).

Now, we give an example to illustrate that the converse of the property (ii) in Theorem 2.2 is not true in general.

Example 2.3. Let $S=[-1,0], \alpha=4$. Let $F_{1}, F_{2}: \mathbb{R} \rightarrow \mathbb{R}$ be defined by

$$
F_{1}(x)=x+1, \quad F_{2}(x)=x .
$$

We have

$$
\left(F_{1}(x)(y-x), F_{2}(x)(y-x)\right)=((x+1)(y-x), x(y-x))
$$

For $x=0$,

$$
\left(F_{1}(x)(y-x), F_{2}(x)(y-x)\right)=((y-0), 0) \notin-i n t \mathbb{R}_{+}^{2}, \quad \forall y \in S,
$$

which implies that $0 \in \operatorname{Sol}(F, S)$. However,

$$
\theta(0)=\max _{\lambda \in \Lambda} \max _{y \in S}\left\{(0-y)\left(\lambda_{1} F_{1}(0)+\lambda_{2} F_{2}(0)\right)-2\|y-0\|^{2}\right\}=\max _{\lambda \in \Lambda} \max _{y \in S}\left\{\lambda_{1}(0-y)-2\|y-0\|^{2}\right\} .
$$


For $\lambda=\left(\lambda_{1}, \lambda_{2}\right)=(1,0)$,

$$
\max _{y \in S}\left\{\lambda_{1}(0-y)-2\|y-0\|^{2}\right\}=\frac{1}{8}
$$

Thus, we have

$$
\theta(0)=\max _{\lambda \in \Lambda} \max _{y \in S}\left\{\lambda_{1}(0-y)-2\|y-0\|^{2}\right\} \geqslant \frac{1}{8},
$$

which implies that the converse of the property (ii) in Theorem 2.2 does not hold.

\section{Robust weighted expected residual minimization model}

In the following we mainly discuss SVVI: Find $x^{*} \in S$ such that

$$
\left(\left(y-x^{*}\right)^{\mathrm{T}} \mathrm{F}_{1}\left(x^{*}, \xi\right), \cdots,\left(y-x^{*}\right)^{\mathrm{T}} \mathrm{F}_{\mathrm{m}}\left(x^{*}, \xi\right)\right) \notin-\operatorname{int} \mathbb{R}_{+}^{\mathrm{m}}, \quad \forall y \in S \text {, a.e. } \xi \in \Xi,
$$

where $S$ is a nonempty, convex, and closed set in $\mathbb{R}^{n}, \Xi \subset \mathbb{R}^{r}$ denotes the support of the random variable $\xi$, and 'a.e.' is the abbreviation for "almost every".

Similarly to the preceding discussion, for any given $\lambda \in \Lambda$, consider SVI as follows: Find $x^{*} \in S$ such that

$$
\left(y-x^{*}\right)^{T} \sum_{j=1}^{m} \lambda_{j} F_{j}\left(x^{*}, \xi\right) \geqslant 0, \quad \forall y \in S, \quad \text { a.e. } \quad \xi \in \Xi .
$$

In general, it cannot be expected that problem (3.1) has a common solution for almost every $\xi \in \Xi$. Therefore, for the sake of getting reasonable solutions in some senses, a suitable deterministic model for problem (3.1) becomes an important topic. In what follows, we assume that $\alpha$ is a positive parameter. For any given $\lambda \in \Lambda$, the regularized gap function $\phi$ is defined for SVI (3.1) as follows:

$$
\phi(x, \lambda, \xi):=\max _{y \in S}\left\{(x-y)^{\mathrm{T}} \sum_{j=1}^{m} \lambda_{j} F_{j}(x, \xi)-\frac{\alpha}{2}\|x-y\|^{2}\right\} .
$$

Then, for given $\lambda \in \Lambda$, for any $x \in S$ and any $\xi \in \Xi$, we have

$$
\phi(x, \lambda, \xi)=(x-H(x, \lambda, \xi))^{T} \sum_{j=1}^{m} \lambda_{j} F_{j}(x, \xi)-\frac{\alpha}{2}\|x-H(x, \lambda, \xi)\|^{2},
$$

where

$$
H(x, \lambda, \xi):=\operatorname{Proj}_{S}\left(x-\alpha^{-1} \sum_{j=1}^{m} \lambda_{j} F_{j}(x, \xi)\right) .
$$

Motivated by the works [3, 22] on SVI, the robust weighted ERM model for SVVI is proposed as follows:

$$
\min _{x \in S} \max _{\lambda \in \Lambda} \mathbb{E}[\phi(x, \lambda, \xi)]
$$

where $\mathbb{E}$ denotes the mathematical expectation.

Since problem (3.3) involves mathematical expectation in the objective function, it is generally difficult to evaluate exactly or these integrals cannot be calculated in a closed form, we apply SAA techniques to approximate the expectation.

In general, for an integrable function $\psi: \Xi \rightarrow \mathbb{R}$, the sample average $\frac{1}{N} \sum_{i=1}^{N} \psi\left(\xi^{i}\right)$ is employed to approximate the expected value $\mathbb{E}[\psi(\xi)]$. By the strong law of large numbers, we have the following result. 
Lemma 3.1. Assume that $\psi: \Xi \rightarrow \mathbb{R}$ is an integrable function, then

$$
\lim _{N \rightarrow \infty} \frac{1}{N} \sum_{i=1}^{N} \psi\left(\xi^{i}\right)=\mathbb{E}[\psi(\xi)]
$$

holds with probability one, where $\xi^{1}, \cdots, \xi^{\mathrm{N}}$ are independently and identically distributed samples of $\xi$.

Thus, let $\xi^{1}, \cdots, \xi^{N}$ be an independent and identically distributed sampling of $\xi$, then the approximation problem of (3.3) as follows:

$$
\min _{x \in S} \max _{\lambda \in \Lambda} \frac{1}{N} \sum_{i=1}^{N} \phi\left(x, \lambda, \xi^{i}\right) .
$$

Next we list some assumptions which are needed in the following section.

(A1) For any $x \in S$ and almost every $\xi \in \Xi$, there exists a measurable function $\kappa(\xi)$ such that

$$
\sum_{j=1}^{m}\left\|F_{j}(x, \xi)\right\| \leqslant k(\xi)
$$

with $\mathbb{E}\left[\kappa^{2}(\xi)\right]<+\infty$.

(A2) There exists a nonnegative measurable function $C(\xi)$ such that $\mathbb{E}\left[C^{2}(\xi)\right]<+\infty$ and

$$
\left\|F_{j}(y, \xi)-F_{j}(x, \xi)\right\| \leqslant C(\xi)\|y-x\|, \quad \forall x, y \in S, j=1, \cdots, m
$$

for all $\xi \in \Xi$, and $F_{j}$ is measurable in $\xi$ for every $x \in S$.

Definition 3.2 ([25]). It is said that the function $\mathrm{H}(x, \xi)$ is random lower semicontinuous if the associated epigraphical multifunction $\xi \mapsto \operatorname{epiH}(\cdot, \xi)$ is closed valued and measurable.

Theorem 3.3. Assume that conditions (A1) and (A2) are satisfied. Then, $\phi(x, \lambda, \cdot)$ is measurable for every $(x, \lambda) \in$ $\mathrm{S} \times \Lambda$.

Proof. Since $F_{j}(\cdot, \xi)$ is continuous for almost every $\xi \in \Xi$, and $F_{j}(x, \cdot)$ is measurable for every $x \in S$, then, $\sum_{j=1}^{m} \lambda_{j} F_{j}(x, \xi)$ is continuous in $(x, \lambda)$ for almost every $\xi \in \Xi$, and $\sum_{j=1}^{m} \lambda_{j} F_{j}(x, \cdot)$ is measurable for every $(x, \lambda) \in S \times \Lambda$. This implies that the function

$$
(x-y)^{\mathrm{T}} \sum_{j=1}^{m} \lambda_{j} F_{j}(x, \xi)-\frac{\alpha}{2}\|x-y\|^{2}
$$

is continuous in $(x, y, \lambda)$ for almost every $\xi \in \Xi$ and measurable in $\xi$ for every $(x, y, \lambda) \in S \times S \times \Lambda$. That is, the above function is random lower semicontinuous. Therefore, the measurability of $\phi(x, \lambda, \xi)$ follows from Theorem 7.37 of [25].

We first discuss the convergence of the optimal value and the set of optimal solutions of the approximate problem (3.4). We let $\theta^{*}$ and $S^{*} \subset S$ be the optimal value and the set of optimal solutions of (3.3), respectively. Let $\theta_{\mathrm{N}}$ and $S_{\mathrm{N}}^{*} \subset S$ be the optimal value and the set of optimal solutions of (3.4), respectively.

Theorem 3.4. Let $\mathrm{S}$ be a compact set. Suppose that conditions (A1) and (A2) hold. Then, $\theta_{\mathrm{N}} \rightarrow \theta^{*}$ and $\mathbb{D}\left(S_{N}^{*}, S^{*}\right) \rightarrow 0$ as $\mathrm{N} \rightarrow \infty$. 
Proof. It follows from assumption (A2) and the compactness of $S$ that for any $(x, \lambda) \in S \times \Lambda, \phi(x, \lambda, \xi)$ is continuous at $(x, \lambda)$ for a.e. $\xi \in \Xi$. Since $\phi(x, \lambda, \xi) \geqslant 0$ for any $(x, \lambda) \in S \times \Lambda$ and $\xi \in \Xi$, we have from (3.2) that

$$
\begin{aligned}
\frac{\alpha}{2}\|x-H(x, \lambda, \xi)\|^{2} \leqslant(x-H(x, \lambda, \xi))^{T} \sum_{j=1}^{m} \lambda_{j} F_{j}(x, \xi) & \leqslant\|x-H(x, \lambda, \xi)\| \sum_{j=1}^{m} \lambda_{j}\left\|F_{j}(x, \xi)\right\| \\
& \leqslant\|x-H(x, \lambda, \xi)\| \sum_{j=1}^{m}\left\|F_{j}(x, \xi)\right\| .
\end{aligned}
$$

Then,

$$
\|x-H(x, \lambda, \xi)\| \leqslant \frac{2}{\alpha} \sum_{j=1}^{m}\left\|F_{j}(x, \xi)\right\| .
$$

Thus, we have

$$
\begin{aligned}
\phi(x, \lambda, \xi) & =(x-H(x, \lambda, \xi))^{\mathrm{T}} \sum_{j=1}^{m} \lambda_{j} F_{j}(x, \xi)-\frac{\alpha}{2}\|x-H(x, \lambda, \xi)\|^{2} \\
& \leqslant\|x-H(x, \lambda, \xi)\| \sum_{j=1}^{m}\left\|F_{j}(x, \xi)\right\|+\frac{\alpha}{2}\|x-H(x, \lambda, \xi)\|^{2} \leqslant \frac{4}{\alpha}\left(\sum_{j=1}^{m}\left\|F_{j}(x, \xi)\right\|\right)^{2},
\end{aligned}
$$

which implies that $\phi(x, \lambda, \xi)$ is dominated by an integrable function from the assumption (A1). Therefore, it follows from [25, Theorem 7.48] that $\frac{1}{N} \sum_{i=1}^{N} \phi\left(x, \lambda, \xi^{i}\right)$ converges to $\mathbb{E}[\phi(x, \lambda, \xi)]$ with probability one uniformly on $S \times \Lambda$. That is,

$$
\sup _{(x, \lambda) \in S \times \Lambda}\left|\frac{1}{N} \sum_{i=1}^{N} \phi\left(x, \lambda, \xi^{i}\right)-\mathbb{E}[\phi(x, \lambda, \xi)]\right| \rightarrow 0,
$$

with probability one as $N \rightarrow \infty$. Let $\tau_{N}(x):=\max _{\lambda \in \Lambda} \frac{1}{N} \sum_{i=1}^{N} \phi\left(x, \lambda, \xi^{i}\right)$ and $\tau(x):=\max _{\lambda \in \Lambda} \mathbb{E}[\phi(x, \lambda, \xi)]$. Then, we have

$$
\left|\theta_{N}-\theta^{*}\right| \leqslant \max _{x \in S}\left|\tau_{N}(x)-\tau(x)\right| \leqslant \sup _{(x, \lambda) \in S \times \Lambda}\left|\frac{1}{N} \sum_{i=1}^{N} \phi\left(x, \lambda, \xi^{i}\right)-\mathbb{E}[\phi(x, \lambda, \xi)]\right| .
$$

It follows that $\theta_{N} \rightarrow \theta^{*}$ with probability one as $N \rightarrow \infty$.

Based on the above discussions, we have $\mathbb{E}[\phi(x, \lambda, \xi)]$ and $\frac{1}{N} \sum_{i=1}^{N} \phi\left(x, \lambda, \xi^{i}\right)$ are continuous on $S \times \Lambda$. Since $\Lambda$ is compact, this implies that $\tau_{N}(x)$ and $\tau(x)$ are continuous on $S$. Consequently, due to the compactness of $S$, the sets $S^{*}$ and $S_{N}^{*}$ are nonempty with probability one. Suppose that $\mathbb{D}\left(S_{N}^{*}, S^{*}\right) \not \rightarrow 0$. Then, there exists $x_{N} \in S_{N}^{*}$ such that $d\left(x_{N}, S^{*}\right) \geqslant \epsilon$ for some $\epsilon>0$. Since $S$ is compact, we have $x_{N} \rightarrow x^{*} \in S$ (taking a subsequence if necessary). It follows that $x^{*} \notin S^{*}$ and $\tau\left(x^{*}\right)>\theta^{*}$. Since $x_{N} \in S_{N}^{*}$, then $\theta_{N}=\tau_{N}\left(x_{N}\right)$ and

$$
\tau_{N}\left(x_{N}\right)-\tau\left(x^{*}\right)=\tau_{N}\left(x_{N}\right)-\tau\left(x_{N}\right)+\tau\left(x_{N}\right)-\tau\left(x^{*}\right) .
$$

It follows from (3.5)-(3.6) and the continuity of $\tau$ that $\theta_{N}=\tau_{N}\left(x_{N}\right) \rightarrow \tau\left(x^{*}\right)>\theta^{*}$, which leads to a contradiction.

The following results show that exponential convergence rates of the optimal value and the set of optimal solutions of the approximate problem (3.4). 
Theorem 3.5. Let $\mathrm{S}$ be a compact set. Assume that conditions (A1)-(A2) and the following conditions are satisfied:

(i) For all $(x, \lambda) \in S \times \Lambda$, the moment generating function $\mathbb{E}\left[e^{t(\phi(x, \lambda, \xi)-\mathbb{E}[\phi(x, \lambda, \xi)])}\right]$ is finite valued for all $\mathrm{t}$ in a neighborhood of zero;

(ii) For all $\mathrm{t}$ in a neighborhood of zero, $\mathbb{E}\left[\mathrm{e}^{\mathrm{t}(\tilde{\mathrm{\kappa}}(\xi)-\mathbb{E}[\tilde{\mathrm{k}}(\xi)])}\right]$ is finite valued, where $\tilde{\mathrm{\kappa}}(\xi):=\kappa(\xi)\left(6+\frac{5}{\alpha} \mathrm{C}(\xi)+\right.$ $\left.\frac{5}{\alpha} \kappa(\xi)\right)$.

Then, for any $\varepsilon>0$, there exist positive constants $\mathrm{C}(\varepsilon)$ and $\beta(\varepsilon)$ such that

$$
\operatorname{Prob}\left\{\left|\theta_{N}-\theta^{*}\right| \geqslant \varepsilon\right\} \leqslant C(\varepsilon) e^{-N \beta(\varepsilon)}
$$

for sufficiently large $\mathrm{N}$.

Proof. Under the conditions (A1) and (A2), we have

$$
\left|\phi\left(x^{\prime}, \lambda^{\prime}, \xi\right)-\phi(x, \lambda, \xi)\right| \leqslant \tilde{\kappa}(\xi)\left(\left\|x^{\prime}-x\right\|+\left\|\lambda^{\prime}-\lambda\right\|\right), \quad \forall x, x^{\prime} \in S, \lambda, \lambda^{\prime} \in \Lambda
$$

and $\mathbb{E}[\tilde{\mathrm{k}}(\xi)]<\infty$.

From conditions (i)-(ii) and (3.7), it follows by virtue of [26, Theorem 5.1], for any $\varepsilon>0$, there exist positive constants $C(\varepsilon)$ and $\beta(\varepsilon)$ such that

$$
\operatorname{Prob}\left\{\sup _{(x, \lambda) \in S \times \Lambda}\left|\frac{1}{N} \sum_{i=1}^{N} \phi\left(x, \lambda, \xi^{i}\right)-\mathbb{E}[\phi(x, \lambda, \xi)]\right| \geqslant \varepsilon\right\} \leqslant C(\varepsilon) e^{-N \beta(\varepsilon)}
$$

for sufficiently large N. Note that

$$
\begin{aligned}
\left|\theta_{N}-\theta^{*}\right| & =\left|\min _{x \in S} \max _{\lambda \in \Lambda}\left(\frac{1}{N} \sum_{i=1}^{N} \phi\left(x, \lambda, \xi^{i}\right)\right)-\min _{x \in S} \max _{\lambda \in \Lambda} \mathbb{E}[\phi(x, \lambda, \xi)]\right| \\
& \leqslant \max _{x \in S}\left|\max _{\lambda \in \Lambda}\left(\frac{1}{N} \sum_{i=1}^{N} \phi\left(x, \lambda, \xi^{i}\right)\right)-\max _{\lambda \in \Lambda} \mathbb{E}[\phi(x, \lambda, \xi)]\right| \\
& \leqslant \max _{(x, \lambda) \in S \times \Lambda}\left|\frac{1}{N} \sum_{i=1}^{N} \phi\left(x, \lambda, \xi^{i}\right)-\mathbb{E}[\phi(x, \lambda, \xi)]\right| .
\end{aligned}
$$

From (3.8) and (3.9), we have

$$
\operatorname{Prob}\left\{\left|\theta_{\mathrm{N}}-\theta^{*}\right| \geqslant \varepsilon\right\} \leqslant C(\varepsilon) e^{-\mathrm{N} \beta(\varepsilon)} .
$$

Theorem 3.6. Suppose that all conditions of Theorem 3.5 are satisfied. Then, for any $\varepsilon>0$, there exist positive constants $C_{1}(\varepsilon)$ and $\beta_{1}(\varepsilon)$ such that

$$
\operatorname{Prob}\left\{\mathbb{D}\left(S_{N}^{*}, S^{*}\right) \geqslant \varepsilon\right\} \leqslant C_{1}(\varepsilon) e^{-\mathrm{N} \beta_{1}(\varepsilon)}
$$

for sufficiently large N. Moreover, if problem (3.3) satisfies the second order growth condition at $\mathrm{S}^{*}$ :

$$
\max _{\lambda \in \Lambda} \mathbb{E}[\phi(x, \lambda, \xi)] \geqslant \min _{x \in S}\left(\max _{\lambda \in \Lambda} \mathbb{E}[\phi(x, \lambda, \xi)]\right)+K d\left(x, S^{*}\right), \forall x \in S,
$$

where $\mathrm{K}$ is a positive constant, then $\mathrm{C}_{1}(\varepsilon)=\mathrm{C}\left(\frac{1}{3} \mathrm{~K} \varepsilon^{2}\right)$ and $\beta_{1}(\varepsilon)=\beta\left(\frac{1}{3} \mathrm{~K} \varepsilon^{2}\right)$ where $\mathrm{C}(\varepsilon)$ and $\beta(\varepsilon)$ are given in (3.8).

Proof. By Lemma 3.8 of [20], we have for any $\varepsilon>0$, there exists $\delta(\varepsilon)$ such that

$$
\mathbb{D}\left(\mathrm{S}_{\mathrm{N}}^{*}, \mathrm{~S}^{*}\right) \leqslant \varepsilon,
$$

if $\max _{x \in S}\left|\max _{\lambda \in \Lambda} \frac{1}{N} \sum_{i=1}^{N} \phi\left(x, \lambda, \xi^{i}\right)-\max _{\lambda \in \Lambda} \mathbb{E}[\phi(x, \lambda, \xi)]\right| \leqslant \delta(\varepsilon)$. Therefore, we have

$$
\operatorname{Prob}\left\{\mathbb{D}\left(S_{N}^{*}, S^{*}\right) \geqslant \varepsilon\right\} \leqslant \operatorname{Prob}\left\{\max _{x \in S}\left|\max _{\lambda \in \Lambda} \frac{1}{N} \sum_{i=1}^{N} \phi\left(x, \lambda, \xi^{i}\right)-\max _{\lambda \in \Lambda} \mathbb{E}[\phi(x, \lambda, \xi)]\right| \geqslant \delta(\varepsilon)\right\} .
$$


It follows from (3.8)-(3.9) and (3.11) that there exist positive constants $C_{1}(\varepsilon)$ and $\beta_{1}(\varepsilon)$ such that

$$
\operatorname{Prob}\left\{\mathbb{D}\left(S_{N}^{*}, S^{*}\right) \geqslant \varepsilon\right\} \leqslant C_{1}(\varepsilon) e^{-N \beta_{1}(\varepsilon)}
$$

for sufficiently large $\mathrm{N}$.

If the condition (3.10) holds, we have the result from Theorem 3.10 of [20] immediately.

\section{Acknowledgment}

The authors are grateful to the editor and two anonymous referees for valuable comments and suggestions to improve the paper. This work was supported in part by the National Natural Science Foundation of China (11301571, 11471059), the Basic and Advanced Research Project of Chongqing (cstc2017jcyjAX0382, cstc2015jcyjBX0131), the Program for University Innovation Team of Chongqing (CXTDX201601022) and the Education Committee Project Foundation of Bayu Scholar.

\section{References}

[1] C. Charitha, J. Dutta, Regularized gap functions and error bounds for vector variational inequalities, Pac. J. Optim., 6 (2010), 497-510. 1

[2] C. Charitha, J. Dutta, C. S. Lalitha, Gap functions for vector variational inequalities, Optimization, 64 (2015), 1499_ 1520. 1

[3] X.-J. Chen, M. Fukushima, Expected residual minimization method for stochastic linear complementarity problems, Math. Oper. Res., 30 (2005), 1022-1038. 1, 3

[4] G.-Y. Chen, X.-X. Huang, X.-Q. Yang, Vector optimization, Set-valued and variational analysis, Lecture Notes in Economics and Mathematical Systems, Springer-Verlag, Berlin, (2005). 1

[5] X.-J. Chen, G.-H. Lin, CVaR-based formulation and approximation method for stochastic variational inequalities, Numer. Algebra Control Optim., 1 (2011), 35-48. 1

[6] X.-J. Chen, R. J.-B. Wets, Y.-F. Zhang, Stochastic variational inequalities: residual minimization smoothing sample average approximations, SIAM J. Optim., 22 (2012), 649-673. 1

[7] X.-J. Chen, C. Zhang, M. Fukushima, Robust solution of monotone stochastic linear complementarity problems, Math. Program., 117 (2009), 51-80. 1

[8] F. Facchinei, J.-S. Pang, Finite-dimensional variational inequalities and complementarity problems, Vol. I, II, Springer Series in Operations Research, Springer-Verlag, New York, (2003). 1

[9] M. Fukushima, Equivalent differentiable optimization problems and descent methods for asymmetric variational inequality problems, Math. Programming, 53 (1992), 99-110. 2, 2

[10] F. Giannessi, Theorems of alternative, quadratic programs and complementarity problems, Variational inequalities and complementarity problems, Proc. Internat. School, Erice, (1978), Wiley, Chichester, (1980), 151-186. 1

[11] F. Giannessi (ed.), Vector variational inequalities and vector equilibria, Mathematical theories, Nonconvex Optimization and its Applications, Kluwer Academic Publishers, Dordrecht, (2000). 1

[12] G. Gürkan, A. Y. Özge, S. M. Robinson, Sample-path solution of stochastic variational inequalities, Math. Program., 84 (1999), 313-333. 1

[13] J. Hu, T. Homem-de-Mello, S. Mehrotra, Multi-criterion robust and stochastic dominance-constrained models with application to budget allocation in homeland security, Manuscript, (2010). 1, 2

[14] J. Hu, S. Mehrotra, Robust and stochastically weighted multiobjective optimization models and reformulations, Oper. Res., 60 (2011), 936-953. 1, 2

[15] N. J. Huang, J. Li, X. Q. Yang, Weak sharpness for gap functions in vector variational inequalities, J. Math. Anal. Appl., 394 (2012), 449-457. 1

[16] H.-Y. Jiang, H.-F. Xu, Stochastic approximation approaches to the stochastic variational inequality problem, IEEE Trans. Automat. Control, 53 (2008), 1462-1475. 1

[17] G. M. Lee, D. S. Kim, B. S. Lee, N. D. Yen, Vector variational inequality as a tool for studying vector optimization problems, Nonlinear Anal., 34 (1998), 745-765. 1, 2.1

[18] G.-H. Lin, X. Chen, M. Fukushima, New restricted NCP functions and their applications to stochastic NCP and stochastic MPEC, Optimization, 56 (2007), 641-953. 1

[19] G.-H. Lin, M. Fukushima, Stochastic equilibrium problems and stochastic mathematical programs with equilibrium constraints: a survey, Pac. J. Optim., 6 (2010), 455-482. 1

[20] Y.-C. Liu, H.-F. Xu, Stability analysis of stochastic programs with second order dominance constraints, Math. Program., 142 (2013), 435-460. 3, 3

[21] M.-J. Luo, G.-H. Lin, Convergence results of the ERM method for nonlinear stochastic variational inequality problems, J. Optim. Theory Appl., 142 (2009), 569-581. 1 
[22] M.-J. Luo, G.-H. Lin, Expected residual minimization method for stochastic variational inequality problems, J. Optim. Theory Appl., 140 (2009), 103-116. 3

[23] M.-J. Luo, G.-H. Lin, Stochastic variational inequality problems with additional constraints and their applications in supply chain network equilibria, Pac. J. Optim., 7 (2011), 263-279. 1

[24] R. T. Rockafellar, R.J.-B. Wets, Stochastic variational inequalities: single-stage to multistage, Math. Program., 165 (2017), 331-360. 1

[25] A. Shapiro, D. Dentcheva, A. Ruszczyński, Lectures on stochastic programming, Modeling and theory, MPS/SIAM Series on Optimization, Society for Industrial and Applied Mathematics (SIAM), Philadelphia, PA; Mathematical Programming Society (MPS), Philadelphia, PA, (2009). 3.2, 3, 3

[26] A. Shapiro, H.-F. Xu, Stochastic mathematical programs with equilibrium constraints, modelling and sample average approximation, Optimization, 57 (2008), 395-418. 3

[27] M. Sofonea, Y.-B. Xiao, Fully history-dependent quasivariational inequalities in contact mechanics, Appl. Anal., 95 (2016), 2464-2484. 1

[28] Y.-B. Xiao, N.-J. Huang, Y. J. Cho, A class of generalized evolution variational inequalities in Banach spaces, Appl. Math. Lett., 25 (2012), 914-920.

[29] Y.-B. Xiao, X.-M. Yang, N.-J. Huang, Some equivalence results for well-posedness of hemivariational inequalities, J. Global. Optim., 61 (2015), 789-802. 1

[30] Y. Xie, U. V. Shanbhag, On robust solutions to uncertain linear complementarity problems and their variants, SIAM J. Optim., 26 (2016), 2120-2159. 1

[31] H.-F. Xu, Sample average approximation methods for a class of stochastic variational inequality problems, Asia-Pac. J. Oper. Res., 27 (2010), 103-119. 1

[32] X. M. Yang, X. Q. Yang, K. L. Teo, Some remarks on the Minty vector variational inequality, J. Optim. Theory Appl., 121 (2004), 193-201. 1

[33] Y. Zhao, J. Zhang, X.-M. Yang, G.-H. Lin, Expected Residual Minimization Formulation for a Class of Stochastic Vector Variational Inequalities, J. Optim. Theory Appl., 175 (2017), 545-566. 1

[34] Y.-F. Zhang, X.-J. Chen, Regularizations for stochastic linear variational inequalities, J. Optim. Theory Appl., 163 (2014), 460-481. 1 